\title{
El patrimonio de marca (brand equity) en el sector de la educación: un estudio exploratorio en el segmento de cursos de MBA
}

\author{
Edson Crescitelli \\ Universidad de São Paulo \\ São Paulo, Brasil \\ Tomislav Mandakovic \\ Escuela de Negocios, Barry University \\ Miami Shores, FL
}

\section{RESUMEN:}

Las escuelas de negocios se enfrentan a una fuerte competencia en la disputa por estudiantes, dado que la marca es una alternativa eficaz para mejorar la competitividad de las instituciones. Este estudio tiene como objetivo identificar los factores determinantes en la formación de imagen de marca en el sector de la educación. El método utilizado fue exploratorio, incluida la investigación bibliográfica y de campo con más de 450 estudiantes de MBA de Brasil. Los resultados indican que la imagen de las escuelas de negocios es positiva, pero ellas aún no cuentan con un proceso de construcción del patrimonio de marca (brand equity) adecuado a las necesidades impuestas por un mercado cada vez más competitivo.

Palabras clave: sector de la educación, las escuelas de negocios, MBA, marca y patrimonio de marca (brand equity).

\section{ABSTRACT:}

Business schools have become an extremely competitive market in their effort to attract students. In any competitive context, brand equity becomes an increasingly important management tool for even these organizations. This study aims to identify the determinant factors of the brand equity in the sector of MBA programs. Given the exploratory nature of the study, the investigative method involved a review of the literature supplemented by a survey of over 450 MBA students from representative business schools in Brazil. In general, the results indicate that the image of business schools is positive but they have not yet designed a process to develop their own Patrimony of Mark (brand equity) that satisfies the needs imposed by an increasingly competitive market.

Keywords: education sector, business schools, MBA, brand and brand equity. 


\section{INTRODUCCIÓN}

El sector de la educación es un tema de interés para la sociedad; posee características peculiares, como las acciones de los gobiernos (de vigilancia y la prestación de servicios de educación), y el seguimiento de los distintos tipos de organizaciones que representan a la sociedad civil (medios de comunicación, organizaciones no gubernamentales y asociaciones). Sin embargo, aunque presenten características peculiares, algunos de sus segmentos están más sujetos a las reglas del mercado, tales como las escuelas de negocios y sus programas de MBA (Master of Business Administration). Estos, a pesar de pertenecer al sector de la educación, tienen también muchas características del sector empresarial, especialmente en la competitiva lucha por atraer estudiantes. Teniendo en cuenta este hecho, es innegable que las escuelas necesitan desarrollar estrategias competitivas para lograr sus objetivos y alcanzar el éxito. Por esta razón, este segmento será el foco de este trabajo.

En este contexto competitivo, la marca se convierte, cada vez más, en una importante herramienta de gestión para organizaciones de cualquier tamaño y sector. El valor de marca es una de las alternativas más eficaces para mejorar la competitividad de las instituciones (Ries y Ries, 2006) ya que puede generar diferencias entre las organizaciones que operan en mercados competitivos (Kapferer, 2003; Haig, 2003). Por lo tanto, dado este escenario, este estudio se propuso identificar los factores que influyen en la percepción del valor de marca en el segmento de los cursos de MBA.

Teniendo en cuenta la naturaleza exploratoria del estudio, el método empleado consistió en la investigación y la revisión de la literatura relevante para el tema, complementado por una investigación de campo con más de 450 cursos para estudiantes de MBA de diferentes e importantes escuelas de negocios en Brasil.

\section{REVISIÓN DE LA LITERATURA}

La marca se define por la American Marketing Association (AMA) como "un nombre, término, signo, símbolo o diseño o una combinación de todo esto, para identificar productos o servicios de un 
proveedor o un grupo de proveedores de distinguir entre los otros competidores". La función de la marca en el contexto de la comercialización ha evolucionado considerablemente desde su aparición. Hoy día, además de identificar un producto y distinguirlo de otros productos competidores - como era su función original -la marca añade valor al producto, ampliando su potencial competitivo, como pone de relieve Aaker (2003): "[s]i una marca no hace esfuerzos para desarrollar o mantener la diferenciación, los consumidores no tendrán ninguna base para la elección de la marca sobre las demás”. Otro factor representante de la posición de relieve ocupada por la marca es que, aunque no aparezca explícitamente en el balance de las empresas, la mayoría de los administradores estarían de acuerdo con la afirmación de que la marca de una empresa es uno de sus más importantes activos intangibles (Schultz, 2004).

La marca es un factor importante para la competitividad de las organizaciones, ya que los clientes no sólo compran productos o servicios, sino también el valor de su marca. Según Hoefler y Keller (2003), "los consumidores deciden sus compras basados en los factores que ellos creen ser importantes, o sea, las marcas que tienen más valor añadido". La importancia del valor de la marca se destaca por Shultz (2001) quien argumenta que la promesa de la marca o propuesta de valor no es un palo, un icono o un color o un elemento gráfico, a pesar de todos estos aspectos pueden contribuir. Más bien, es el corazón y el alma de la marca. El valor de marca es el potencial de ésta, es decir, su capacidad de ser conocida, reconocida por el mercado y generar valor añadido y diferencial para el producto (Belch y Belch, 2003; Shimp, 1997; Clow y Baack, 2004; Aaker y Joachimsthaler, 2000). El valor de marca puede ser entendido como la suma de los activos tangibles e intangibles de un producto o servicio en particular y puede ser analizado desde dos aspectos diferentes: el valor financiero de la marca (es decir, su valor patromonial, expresado en valor monetario) y el valor emocional de la marca (cuando la misma es admirada y respetada).

En las últimas décadas, varios autores se han dedicado a desarrollar modelos para explicar la formación del valor de marca. Entre los diversos modelos existentes figuran los propuestos por Keller (1998 y 2001), Aaker (1998), Aaker y Joachimsthal (2000), el modelo Bran- 
dz de la consultoría Millward Brown y WPP, y finalmente el modelo BAV - Brand Asset Valuation de los activos de la marca, de la agencia Young \& Rubicam. El concepto de Branding puede considerarse como el acto de generación de marca, o para gestionar el proceso (crear y mantener) el valor de la marca (Martins, 2000; Keller y Machado, 2005). Estos modelos se encuentran entre los más extendidos en el estudio del valor de marca. Considerando que estos modelos tienen mucho más puntos de convergencia que divergencia, se utiliza el modelo de Aaker (1998) como referencia para el planteamiento de los elementos del modelo de valor de marca. Según el autor, la marca se compone de cinco dimensiones: conocimiento de marca (se refiere a la presencia de la marca en la mente de los consumidores); asociaciones de marca (representa lo que la organización quiere que la marca represente en la mente de los clientes); calidad percibida (se encuentra en la esencia de lo que el cliente está comprando, por lo cual está directamente relacionada con la identidad de una marca); lealtad a la marca (incluye cuestiones o aspectos tales como la lealtad del cliente y su disposición a seguir activo en sus relaciones con la organización); y el complemento de otros activos relacionados con la marca (patentes, la respetabilidad en el canal de distribución, nivel de competitividad).

En los últimos tiempos, varias teorías acerca de la marca han sido desarrolladas y mucho ha cambiado a fin de comprender mejor el papel y la importancia de la marca en el contexto de la comercialización. Conceptos tales como: el valor económico de marca, posicionamiento de marcas, la jerarquía de la marca, una extensión de marca, la marca matriz, sub-marcas, co-branding, la arquitectura de marca, identidad de marca, entre otros, parecen ser cada vez más familiares para los profesionales y estudiosos de mercadeo. Sin embargo, dos conceptos son fundamentales para entender la fase actual del estudio de marca y, de alguna manera, incluir a todos los demás. Son los conceptos de marca y de branding. El primero, la marca es el objetivo final que concentra y materializa todos los esfuerzos del proceso de la gestión de la marca. Por lo tanto, se puede considerar que el branding es el proceso de gestión de la marca, para generar equidad de ésta, que a su vez hará que la marca sea más valiosa, potencializando su efecto en el proceso competitivo de mercado. De esta manera, estos 
dos conceptos pueden ser considerados como aspectos complementarios y no como sinónimos, como a menudo son tratados.

\section{Panorama del MBA}

\section{Perfil de los cursos del MBA}

Actualmente hay una gran variedad de cursos en el MBA que promueven la competitividad del sector en los modelos de cursos, los contenidos curriculares y la reputación de las escuelas. El proceso de atraer a los estudiantes se hace cada vez más intenso e internacionalizado, porque la globalización ya no es exclusiva de las empresas que ofrecen productos físicos. En el contexto actual, es evidente en las escuelas de negocios, públicas o privadas, la necesidad de adoptar estrategias competitivas para lograr sus objetivos para lograr el éxito.

Es muy difícil evaluar las escuelas en relación con su prestigio, porque implica muchos factores que no siempre se pueden comparar directamente entre ellos. Uno de los indicadores más utilizados para evaluar la reputación de las escuelas son las clasificaciones publicadas por revistas como se indica en la Tabla 1. Sin embargo, la variación de los resultados refleja que hay un patrón establecido para la formación de tales clasificaciones.

\section{Tabla 1}

\section{CLASIFICACIÓN DE LAS ESCUELAS DE NEGOCIOS}

\begin{tabular}{ccccc}
\hline $\begin{array}{c}\text { Ranking } \\
\text { Position }\end{array}$ & $\begin{array}{c}\text { BusinessWeek } \\
2004 / 2005 \\
\text { (solo E.E.U.U.) }\end{array}$ & $\begin{array}{c}\text { USNews } \\
2005 \\
\text { (only USA) }\end{array}$ & $\begin{array}{c}\text { BusinessWeek } \\
2004 / 2005 \\
\text { (internacional - out USA) }\end{array}$ & $\begin{array}{c}\text { America Economia } \\
2006 \\
\text { (global) }\end{array}$ \\
\hline $1^{\text {ro }}$ & $\begin{array}{c}\text { Northwestern } \\
\text { (Kellog) }\end{array}$ & Harvard & Queens & Stanford \\
$2^{\text {do }}$ & Chicago & Stanford & IMD & $\begin{array}{c}\text { Pennsylvania } \\
\text { (Wharton) }\end{array}$ \\
$3^{\text {ro }}$ & $\begin{array}{c}\text { Pennsylvania } \\
\text { (Wharton) }\end{array}$ & $\begin{array}{c}\text { Pennsylvania } \\
\text { (Wharton) }\end{array}$ & INSEAD & Harvard \\
$4^{\text {to }}$ & $\begin{array}{c}\text { Stanford } \\
\text { Massachusetts } \\
\text { (Sloan) }\end{array}$ & ESADE & INSEAD \\
$5^{\text {to }}$ & Harvard & $\begin{array}{c}\text { Northwestern } \\
\text { (Kellog) }\end{array}$ & Business School & $\begin{array}{c}\text { Massachusetts } \\
\text { (Sloan) }\end{array}$
\end{tabular}

Fuente: Revistas BusinessWeek, USNews y America Economia 
Las cinco escuelas de negocios de América Latina más valoradas de acuerdo con el ranking de la revista América Economía (2006) son las siguientes: el INCAE (Costa Rica / Nicaragua), el ITAM (México), FGV-EAESP (Brasil), Universidad Adolfo Ibáñez (Chile) y la Pontificia Universidad Católica de Chile (Chile).

\section{Panorama de los cursos de MBA}

Tras un descenso en la última década, la demanda de cursos de MBA volvió a crecer en términos de intereses de los estudiantes en las escuelas. Según la Asociación de MBA (AMBA), hay alrededor de 1,500 escuelas de negocios aceptables que ofrecen programas MBA en todo el mundo, y otras tantas por debajo de las normas de calidad que uno esperaría de un proveedor de MBA. El uso de técnicas de mercadeo, servicios de mercadeo y mercadeo de relaciones son herramientas útiles en este proceso y deben ser aplicadas para mejorar la competitividad de las escuelas de negocios (Eccles, 2004; Nguyen Le Blanc, 1999; Mazzarol, 1998 y Nicholls et al, 1995). Para Burton (2002), la competencia en el segmento de MBA tiende a parecerse al segmento corporativo, cuyas técnicas de comercialización son ampliamente utilizadas, contrario a lo que ocurre en el segmento educativo. Sin embargo, su formato y contenido deben estar en constante evolución para satisfacer las necesidades del mercado, con una trayectoria principalmente orientada a la formación de ejecutivos.

A la luz de los nuevos tiempos, las escuelas de negocios pueden desempeñar un papel importante en el desarrollo y la formación de las personas a ser culturalmente sensibles y capaces de trabajar en un entorno diverso y multicultural (Hawawini, 2005). Hay una fuerte tendencia de los cursos de MBA hacia la revisión actual de sus programas (Baruch y Leeming, 1996; Mintzberg, 2004), adaptándose a la nueva orden de los mercados y a las demandas de los estudiantes y de las empresas. Una de estas demandas es que los cursos sean cada vez más globalizados (Brasahaw, 2006), pues los estudiantes formados en estos cursos trabajarán en las empresas que operan en los mercados globalizados (Ewing, 2006). En este contexto más competitivo, la búsqueda de un mayor equilibrio en la relación entre la teoría y la aplicación práctica de los conceptos es una 
tendencia que se debe intensificar (Calder, Tybout, 1999; Baruch, Leeming, 1996 y Carnall, 1995), ya que la demanda del mercado debe seguir dicha dirección. Los estudiantes quieren, cada vez más, que el conocimiento se pueda aplicar de manera eficaz y rápida.

\section{EL VALOR DE MARCA Y EL MERCADO EDUCATIVO}

El valor de marca es particularmente importante en el sector de los servicios debido a las características del mismo. La intangibilidad y la variación en la calidad final del "producto" de valor de marca emitido constituyen una referencia importante para el estudiante al momento de contratar un servicio. A menudo la fuerza del valor de marca es el factor más importante en la decisión de compra, especialmente en los casos de compra de servicios con alto grado de participación, cuantía y duración, como la contratación de los servicios educativos que incluyen el tipo MBA. Por tal motivo, las instituciones que ofrecen este servicio deberían prestar especial atención al valor de marca a través de una cuidadosa vigilancia, no sólo a la fuerza de la marca, sino a todos los factores que contribuyen a su formación, ya sean positivos o negativos. La pérdida del valor de marca en estos segmentos puede ser un duro golpe sobre los resultados de las instituciones y lo que es aún más impresionante, el más competitivo para el segmento. A veces el daño es irreparable para la institución.

\section{ESTUDIO DE CAMPO}

El mercado brasileño para estudios de postgrado es bastante significativo. Hoy día ronda cerca de los 350 programas de postgrado, compuesto por instituciones públicas, privadas y sin fines de lucro que ofrecen varios tipos de programas de postgrado, entre ellos el tipo de MBA, según el Instituto Nacional de Estudios e Investigaciones Educacionales del Ministerio de Educación y Cultura - INEP / MEC (2003). Este tema se presenta en los principales resultados obtenidos a partir de la investigación de campo llevada a cabo entre los meses de marzo y abril de 2006. Las escuelas consideradas en la investigación se eligieron debido a que forman parte de la clasificación nacional de las mejores escuelas de negocios, publicada por la 
revista Você S/A (en las últimas tres ediciones anuales: 2002, 2003 y 2004), una de las clasificaciones más influyentes y reconocidas por los estudiantes y las empresas. Las escuelas incluidas en la encuesta son: Escuela de Negocios de Brasil - BBS, COPPEAD Institute Universidad Federal de Río de Janeiro - COPPEAD, Escola Superior de Propaganda e Mercadeo - ESPM, la Fundação Armando Alvares Penteado - FAAP, la Fundação Dom Cabral - FDC, la Fundação Getúlio Vargas - FGV, la Fundação Instituto de Administração - FIA, Fundace, Instituto Brasileño de Mercado de Capitales - IBMEC, la Universidad Federal de Rio Grande do Sul - UFRGS y la Universidad de Pittsburgh.

Aunque se trata de un estudio de naturaleza exploratoria, se trabaja con una muestra muy diversa con una mayor representación. Por otro lado, se entrevistó personalmente a 457 personas a través de cuestionarios, incluidos los siguientes: posibles alumnos, alumnos actuales y exalumnos, los diferentes tipos de cursos de MBA y cinco importantes escuelas del país (ESPM, FAAP, la FIA, Anhembi Morumbi y Mackenzie), además de los visitantes de una feria internacional de escuelas de negocios celebrada en la ciudad de São Paulo, que atrae a estudiantes de todo el país, diez de ellos interesados en el post-grado.

El perfil básico de los entrevistados de la encuesta es: graduado en Administración de Empresas (44.0\%), tiene hasta 30 años (71.0\%), trabaja como un empleado y ocupa los puestos de dirección de la baja $(60.0 \%)$. Elegimos como objetivo no segmentar los respondientes, puesto que por la propuesta y la naturaleza de la investigación eso no sería pertinente.

\section{Resultados}

El perfil predominante de la muestra es el siguiente: de ambos sexos, graduados en Administración de Empresas, tienen 30 años en promedio, trabajan como empleados y ocupan puestos de gestión más bajos. El área de aumento en la incidencia de interés es el mercadeo. En un universo de 412 respuestas válidas, el $42.0 \%$ indica esta zona como la más interesante, mientras, las más distantes son las áreas de recursos humanos, finanzas, administración y comuni- 
cación. La principal razón dada para seguir el programa MBA, entre las 397 respuestas válidas es el "desarrollo profesional", seguido por el de "desarrollo personal”. Esto indica que el mayor interés es mejorar las competencias en el mercado laboral. En la Tabla 2 se muestran las tres escuelas de negocios nacionales, entre la mayoría mencionada. Se encontró, a base de los resultados, que ninguna de las escuelas obtuvo porcentaje importante. Esto indica que las marcas de las escuelas de negocios no son tan prominentes en la mente de los estudiantes.

Tabla 2

ESCUELAS DE NEGOCIO NACIONALES MENCIONADAS ESPONTÁNEAMENTE

\begin{tabular}{|c|c|c|}
\hline 1a. opción & 2a. opción & 3a. opción \\
& & \\
$34 \%$ - FGV & $41 \%-$ FGV & $24 \%$ - IBMEC \\
$19 \%$ - FIA & $16 \%$ - IBMEC & $15 \%$ - ESPM \\
$16 \%$-ESPM & $12 \%$ - USP & $13 \%-$ FGV \\
\hline
\end{tabular}

FGV = Fundação Getúlio Vargas, FIA = Fundação Instituto de Administração, ESPM = Escola Superior de Propaganda e Mercadeo, IBMEC = Instituto Brasileño de Mercado de Capitales, USP = Universidad de São Paulo.

Para las escuelas internacionales (Tabla 3), Harvard tiene la mayor atención. Otro factor importante es que todas las escuelas que aparecen entre las primeras tres opciones son de América del Norte. Los colegios internacionales son los más citados; éstos se encuentran entre los mejores de la clasificación presentada anteriormente en este trabajo. Sin embargo, los resultados revelan que los estudiantes, en la mayoría de los casos de escuelas internacionales, sólo tenían conocimiento de las marcas de las escuelas de negocios, pues demostraron poco conocimiento acerca de otros importantes atributos de marca y las asociaciones, el área de especialización o líneas de investigación. 
Tabla 3

ESCUELAS INTERNACIONALES MENCIONADAS ESPONTÁNEAMENTE

\begin{tabular}{|c|c|c|}
\hline 1a. opción & 2a. opción & 3a. opción \\
63\% - Harvard & $16 \%$ - Harvard & $11 \%$ - Stanford \\
$7 \%$ - Wharton & $8 \%$ - Pittsburh e MIT & $9,5 \%$ - MIT \\
$3,5 \%$ - Pittsburgh & $7 \%$ - Stanford & $8,5 \%$ - YALE \\
\hline
\end{tabular}

Con respeto a los atributos valorados en una escuela de negocios, el atributo "de calidad del programa" aparece como el más importante. En segundo lugar se menciona "ser reconocido" y en tercer lugar "tiene prestigio". Estos tres atributos están estrechamente relacionados con el valor de marca, lo que refuerza la fuerte influencia del valor de marca de la escuela en el proceso de toma de decisiones de los estudiantes. El orden de los atributos sugiere que deberían ser las prioridades de los directivos en la construcción del valor de marca en una institución educativa.

Utilizando la escala tipo Likert de cinco puntos (totalmente de acuerdo, de acuerdo, neutral, en desacuerdo y totalmente en desacuerdo), los entrevistados opinaron sobre cada una de las once escuelas mejor colocadas en la clasificación utilizada para esta investigación. Las preguntas tenían como base temas de interés al segmento MBA. La Tabla 4 contiene los resultados obtenidos por escuelas. El resultado indica que la actitud de los encuestados varia positivamente con respecto a la forma en que cada escuela se percibe en relación a los atributos enumerados en la columna vertical. Cuanto mayor sea el número, mejor es la relación de la escuela como el atributo. Se percibe una gran variación en los puntos atribuidos a cada una de las escuelas, pero no hay una escuela en particular donde haya una fuerte concentración de las respuestas en un único factor (o un conjunto de factores). Hay notas muy favorables que se perciben, por ejemplo, en la FGV. Sin embargo, aunque en el caso de dicha escuela todas las notas son favorables, no hay un atributo en particular en el que la escuela se ponga de relieve. No puede decirse, por tanto, que esta escuela tiene una fuerte imagen basada en un atributo particular. En contraste, se encontró que todas las escuelas 
muestran una reducción significativa de su promedio en el atributo "tiene buenas alianzas internacionales".

24\% - IBMEC - Instituto Brasileiro de Mercado de Capitales 15\% - ESPM - Escola Superior de Propaganda e Mercadeo 13\% - FGV - Fundação Getulio Vargas

Tabla 4

RANKING DE ESCUELAS POR ATRIBUTO

\begin{tabular}{|c|c|c|c|c|c|c|c|c|c|c|c|}
\hline ESRIBUTOS & BBS & Coppead & ESPM & FAAP & FDC & FGV & FIA & Fundace & IBMEC & UFRGS & Pittsburg \\
\hline $\begin{array}{c}\text { Es uma escuela } \\
\text { tradicional }\end{array}$ & 19 & 41 & $\underline{83}$ & 71 & 37 & $\underline{96}$ & 57 & 10 & 62 & 34 & 40 \\
\hline $\begin{array}{c}\text { tiene buenas } \\
\text { alianzas } \\
\text { intemacionales }\end{array}$ & 25 & 21 & 37 & 22 & 22 & 68 & 38 & 6 & 47 & 16 & 36 \\
\hline $\begin{array}{c}\text { Tiene ex-alumnos de } \\
\text { exito }\end{array}$ & 20 & 37 & 77 & 55 & 31 & $\underline{93}$ & 50 & 7 & 66 & 28 & 33 \\
\hline $\begin{array}{c}\text { Tiene prestigio } \\
\text { (premio/ranking) }\end{array}$ & 22 & 37 & $\underline{84}$ & 53 & 34 & $\underline{96}$ & 56 & 7 & $\underline{80}$ & 24 & 33 \\
\hline $\begin{array}{c}\text { Tiene la excelencia } \\
\text { académica (calidad } \\
\text { de los programas) }\end{array}$ & 20 & 38 & 73 & 47 & 31 & $\underline{92}$ & 54 & 8 & 72 & 30 & 33 \\
\hline $\begin{array}{l}\text { Es reconocida (por } \\
\text { las empresas, los } \\
\text { medios de } \\
\text { comunicación y la } \\
\text { sociedad) }\end{array}$ & 19 & 34 & 74 & 42 & 32 & $\underline{92}$ & 50 & 8 & 63 & 24 & 34 \\
\hline
\end{tabular}

Los resultados indican que, en general, todas las escuelas tienen un bajo porcentaje en todos los atributos. Como no son comparables, es decir, los índices de una escuela no interfieren en las otras, la conclusión es que hay una actitud claramente positiva con respecto a las marcas de las escuelas; aunque con prácticamente ninguno de los atributos que se consideran importantes para una escuela de negocios. La Figura 1 presenta un mapa de percepción construido a 
partir de los datos en la Tabla 4. Una escala tipo Likert se convierte en una escala de 1 a 6 puntos. El mapa se compiló a partir de la media de la Tabla 4, sobre la base de los seis atributos de la izquierda columna vertical. Aunque este procedimiento metodológico no es técnicamente adecuado, se optó por utilizarlo, pues se trata de un estudio exploratorio cuya contribución es relevante para el mismo.

\section{Figura 1}

Mapa de percepción de las escuelas

Derived Stimulus Configuration

Euclidean Distance Model

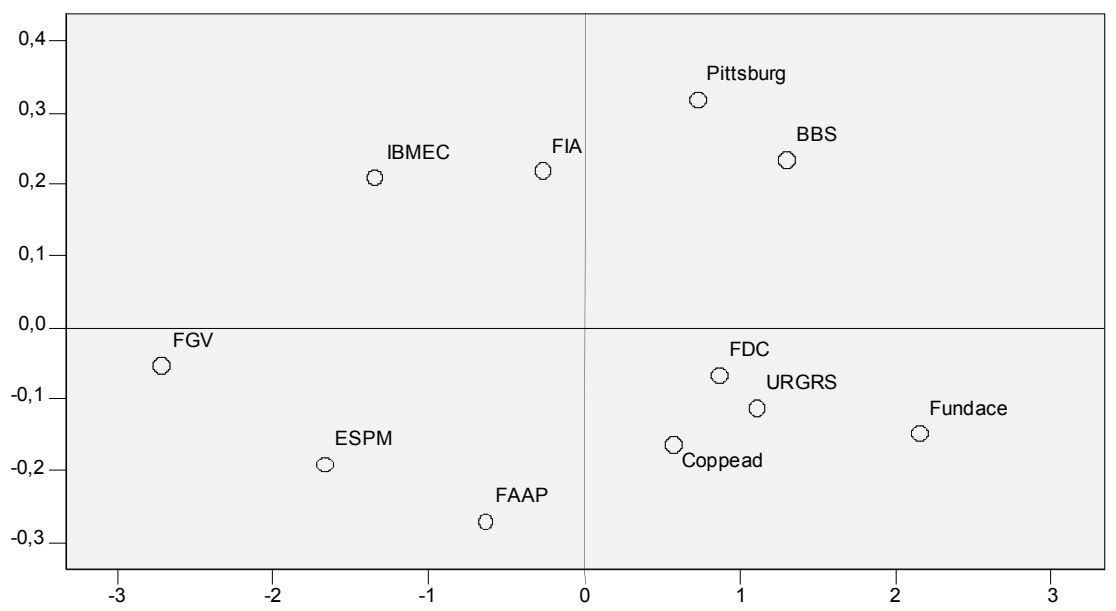

Un análisis de los resultados demuestra que las escuelas forman cuatro grupos bien definidos; cada uno de los grupos ocupa uno de los cuatro cuadrantes de la Figura 1. Esta figura demuestra las escuelas que están más cerca de unos de los cinco atributos considerados más relevantes para la formación de la imagen de una escuela de negocios. Sin embargo, la Figura 1 no indica qué grupo tiene una mejor imagen, pero sí la proximidad entre las escuelas con respecto a su imagen. Se puede encontrar que partir de la disposición señalada en el cuadrante inferior izquierdo, se ubican las escuelas con las mejores puntuaciones en los atributos analizados. En el cuadrante superior izquierdo, se ubican las escuelas que recibieron la media 
más equilibrada entre todos los atributos. En el cuadrante superior derecho, figuran las dos escuelas internacionales que funcionan en el país y en el cuadrante inferior derecho, las escuelas menos conocidas por el público encuestado. No obstante, debe destacarse que esta división de las escuelas de negocios en cuatro cuadrantes no implica que haya cuatro grupos de escuelas claramente vinculados a un atributo en particular. Más bien, como se indicó anteriormente, el mercado no muestra ninguna relación clara de alguna escuela con un atributo específico.

Los dos atributos más valorados por los estudiantes para una escuela de negocios, tal como se indica en la presente investigación, son: la calidad de los programas y el reconocimiento de la institución. Dado que estas dos características están directamente relacionadas con la formación de imagen de marca, tras un análisis de la imagen de las escuelas seleccionadas para estos dos atributos, se puede indicar la actual fortaleza del valor de marca de las marcas de las escuelas encuestadas.

\section{ANÁLISIS de LOS RESULTADOS}

El siguiente análisis fue desarrollado a partir de los fundamentos básicos del valor de marca: conocimiento del valor de marca, marca de asociación y de la calidad percibida y la formación del patrimonio de marca.

Conocimiento del valor de marca - en general se puede constatar que a pesar de todas las escuelas encuestadas aparecen en el clasificación de las mejores escuelas de negocios en el país, no todas tienen el mismo nivel de conocimiento de la marca. Si bien algunas son muy conocidas, otras son prácticamente desconocidas, pero figuran entre las mejores del país. Para las escuelas de negocios internacionales, el nivel de conocimiento del comercio parece bueno, pero limitado a tres escuelas: Harvard, Stanford y Pittsburgh. Cabe señalar que para la Unión Europea y la América Latina, las escuelas, incluso las mejores clasificadas en el ranking, fueron apenas mencionadas por los encuestados, lo que indica que para el conocimiento del valor de marca internacional de escuelas de negocios, sólo las de Estados Unidos se destacan en el país. 
En general, las escuelas nacionales e internacionales con un alto nivel de conciencia (es decir, son muy conocidas) se clasifican entre las mejores en las clasificaciones que miden la calidad de los programas. Sin embargo, el análisis de los datos sugiere que la globalización de la equidad del valor de marca internacional de escuelas de negocios puede no ser un fenómeno tan consolidado como se presume. La baja inversión en comunicación de marca en el país acusado por las escuelas internacionales puede ser en parte la explicación de este fenómeno. Se destaca que la conciencia de marca es el paso esencial para la formación de imagen de marca, más aún cuando se desconoce la marca.

Asociaciones de marca - entre los atributos más valorados por los estudiantes figuran la calidad de los programas, el reconocimiento de la institución y su prestigio. Estos son quizás los principales valores de las marcas a los que las escuelas deben estar estrechamente vinculados. Pero no fue esto lo que el sondeo señaló, a excepción del atributo tradición en el que algunas escuelas están más asociadas a la simple cuestión de acto durante mucho tiempo en el segmento educativo.

Asociaciones con temas como "calidad", "reconocimiento" y "prestigio" serían fundamentales a las asociaciones de base para construir una marca en el segmento, y en la medida en que estas asociaciones se compartan en muchas escuelas, sería necesario buscar la asociación con los otros valores en el segmento para mantener una imagen de marca en el sector diferenciado. Otro punto de atención es la baja importancia que se da al atributo de tipo "es una escuela internacional" y "tener alianzas internacionales". Esto significa que obtener una marca de asociaciones, en consecuencia, no puede ser una de las más eficientes estrategias. Este resultado, agregado a la baja conciencia de las marcas de las escuelas de negocios internacionales, refuerza la idea de que a pesar de todo el proceso de globalización, con la recuperación de las escuelas de negocios internacionales, el segmento de los MBA puede ser un problema para la mayoría de los estudiantes locales.

La calidad percibida - es muy claro que la calidad de los programas MBA es el elemento clave para la construcción del valor de marca. La imagen de las instituciones que se observan a partir de esta prue- 
ba no muestra signos de desgaste, pero ninguna de las marcas está relacionada con este atributo tan destacado.

Formación del Valor de Marca - en ninguna de las escuelas de negocios se presentan indicadores que muestren una imagen de marca y construido. La clara impresión que se da es que ninguno de los esfuerzos para la construcción del valor de marca y la imagen que se forja de dichas esas escuelas se acerca más al resultado de su natural evolución en el tiempo. Dicha imagen está formada principalmente por la difusión a través de los medios impresos (publicidad e informes) y por la comunicación boca a boca (pasar información por medios verbales), más que un proceso planificado de la marca, como hacen las marcas de productos de consumo y las empresas, por ejemplo. Pero sí hay indicadores de que en las escuelas se está construyendo valor de marca. La encuesta también indica que, por lo tanto, se puede considerar el valor de marca como una pizarra en blanco, listo para ser desarrollado.

A base del conjunto de respuestas, podemos deducir que los aspectos más valorados por los estudiantes son: la calidad, el reconocimiento y el prestigio. Estos son los factores que debe reflejar una marca que quiere llegar a ser fuerte en el segmento. El proceso de comunicación con el mercado elaborado por las escuelas, a pesar de ser un aspecto clave en la formación de imagen de marca, hace hincapié en la información sobre programas y cursos, sin explorar el valor de la marca. Ciertamente interfiere con la formación de la imagen de las escuelas.

\section{Consideraciones finales}

Como se indica en la documentación presentada, el valor de marca se refiere esencialmente a factores como el conocimiento de la marca, el valor de marca de las asociaciones y la calidad percibida. En relación con el factor de conocimiento de la marca, los resultados apuntan a una regionalización, es decir, las marcas son bien conocidas a nivel local. Sin embargo, las marcas de otros lugares, aunque bien clasificadas con un alto rango, son poco conocidas. Ya las marcas internacionales, las más prestigiosas entre ellas, cuentan con un buen nivel de reconocimiento por parte de los estudiantes. 
Pero sólo para escuelas de EE.UU., ya que las europeas y latinoamericanas apenas se mencionan en el sondeo. En resumen, las marcas parecen tener una magnitud más regional que nacional.

En el aspecto de las asociaciones de marca, en general en ninguna de ellas parece haber una fuerte asociación con cualquier atributo específico. En un proceso de libre asociación de atributos no se encuentra alguna marca de escuela que esté fuertemente ligada a un atributo específico. En otras palabras, las marcas de las escuelas de negocios no aparecen vinculadas a algún atributo de manera prominente, una posición que podría describirse como neutral. Esta situación, aunque no explícitamente investigada, también pudo constatarse en relación con las escuelas internacionales, porque los encuestados demostraron saber muy poco acerca de ellas, excepto el nombre. Ni siquiera lograron indicar las ciudades de origen a las que pertenecen las universidades.

Sin embargo, cuando la asociación se indujo con la sugerencia de algunos atributos predefinidos, los resultados indicaron que las escuelas tienen una buena reputación, pues ninguna obtuvo una imagen negativa. Aunque no fue objetivo de este proyecto evaluar las razones por las que se presentaron, podemos pensar que esta imagen es el resultado del proceso de comunicación de mercadeo desarrollado por las escuelas de negocios que favorecen la propagación de los cursos y programas, y sólo invierten en acciones de valoración de marca; al menos no en la forma de las empresas en el sector comercial que invierten fuertemente en acciones de comunicación, comercialización de productos y marcas, de diferentes maneras. En general, las instituciones educativas aún no cuentan con un proceso de comunicación para la formación de imagen de marca adecuado a las necesidades impuestas por un mercado cada vez más competitivo. El modelo de comunicación sobre valor de marca adoptado por escuelas de negocios - utilizando medios de comunicación impresos, con anuncios genéricos fundamentalmente sobre el planteamiento del tema, ejecutados sólo durante los períodos de pre-registro - es muy modesto y por debajo de los requisitos para la construcción del valor de marca.

En un estudio de carácter exploratorio, el análisis presentado no puede ser generalizado y se utiliza para entender mejor el fenómeno 
estudiado, teniendo en cuenta sus actuales características. Aunque el mercado de los cursos de MBA tiende a ser muy competitivo, la cuestión del valor de marca en el sector de la educación parece no estar en el mismo nivel de desarrollo que se puede encontrar en el mercado comercial. Considerando que el objetivo inicial era estudiar el comportamiento del valor de marca en el sector de la educación, en particular las escuelas de negocios, se considera que el estudio contribuye a una mejor comprensión del fenómeno. Si bien la investigación de campo se limita al mercado brasileño, se cree que los resultados del estudio pueden ser considerados también para el mercado educativo de las escuelas de negocios en otros países. 


\section{RefERENCIAS}

Aaker, D. A. (1998). Marcas. Brand equity. Gerenciando o valor da marca (2ª ed.). São Paulo: Negócio Editora.

Aaker, D. A. \& Joachimsthaler, E. (2000). Cómo construir marcas líderes. São Paulo: Futura.

Aaker, D. (2003). The power of the branded differentiator. MIT Sloan Management Review, 45 (1), 83-87.

Baruch, Y. \& Leeming, A. (1996). Programming the MBA programme - the quest for curriculum. Journal of Management Development, 15 (7), 27-36.

Belch, G. E. \& Belch, M. A. (2003). Advertising and promotion (6 $6^{\underline{a}}$ ed.). New Jersey: McGraw-Hill.

Brandshaw, D. (2006, 4 de febrero). Escolas de negócios comemoram a volta dos bons tempos. Valor Econômico.

Burton, D. (2002). Consumer educational and service quality: conceptual issues and practical implications. Journal of Services Marketing, 16 (2), 125-142.

Calder, B. J. \& Tybout, A. M. (1999). A vision of theory, research, and the future of business school. Journal of the Academy of Marketing Science, 27 (3), 359-366.

Carnall, C. (1995). The third-generation MBA: global reach and "local" service. The Learning Organization, 2(2), 18-27.

Clow, K. E. \& Baack, D. (2004). Integrated advertising, promotion, and marketing communications (2 ${ }^{\underline{a}}$ ed.). New Jersey: Pearson Prentice Hall.

Eccles, G. (2004). Marketing the corporate university or enterprise academy. The Journal of Workplace Learning, 16(7), 410-418.

Ewing, J. (2006, 13 de marzo). Going global for an MBA. BusinessWeek.

Haig, M. (2003). Brand failures. London: Kogan-Page Limited.

Hawawini, G. (2005). The future of business schools. Journal of Management Development, 24(9), 770-782.

Hoeffler, S. \& Keller, K. L. (2003). The marketing advantages of strong brands. Brand Management, 10(6), 421-445.

Kapferer, J. N. (2003). As marcas. Capital das empresas (3 $3^{\underline{a}}$ ed.). Porto Alegre, Brasil: Bookman. 
Keller, K. L. (1998). Strategic brand management: Building measuring, and managing brand equity. Upper Saddler River, NJ: PrenticeHall.

Keller, K. L. (2001). Building customer-based Brand Equity: A blueprint for creating strong brands. Marketing Management, 10, $15-19$.

Keller, K. L. \& Machado, M. (2005). Gestão estratégica de marcas. São Paulo: Prentice Hall.

Kotler, P. \& Keller, K. L. (2006). Marketing management (12ª ed.). New York: Prentice Hall.

LeBlanc, G. Nguyen, N. (1999). Listening to the customer's voice: Examining perceived service value among business college students. The International Journal of Educational Management, 13(4), 187-198.

Martins, J. R. (2000). Branding: Um manual para você criar, avaliar e gerenciar marcas. São Paulo: Negócio Editora.

Mazzarol, T. (1998). Critical success factors for international education marketing. International Journal of Educational Management, 2(4), 163-175.

Mintzberg, H. (2004). Managers not MBAs. San Francisco: BenettKoehler Publishers Inc.

Nicholls, J., Harris, J., Morgan, E., Clarke, K., \& Sims, D. (1995). Marketing higher education: The MBA experience. International Journal of Educational Management, 9(2), 31-38.

Schultz, D. E. (2004, marzo/abril). Understanding total brand value. Marketing Management, 10-11.

Shimp, T. (1997). Advertising, promotion and supplemental aspects of integrated marketing communications ( $4^{\underline{a}}$ ed.). Orlando, FL: Dryden Press.

Ries, A. \& Ries, L. (2006). A origem das marcas. São Paulo: M Books. 